\title{
INTERACTIONS OF ATOMS WITH HIGH-FIELD SINGLE-CYCLE INFRARED RADIATION
}

\author{
P. H. Bucksbaum and D. You \\ Physics Department, University of Michigan, Ann Arbor, MI 48109-1120 \\ D. R. Dykaar \\ AT\&T Bell Laboratories, Murray Hill, NJ 07974 \\ R. R. Jones \\ Physics Department, University of Virginia, Charlottesville, VA 22903-2458
}

\section{ABSTRACT}

We have studied ionization and excitation of Rydberg atoms by intense sub-picosecond electromagnetic field pulses of less than one cycle duration. The ionization threshold electric field scales as the binding energy (i.e. $\mathrm{n}^{*-2}$ ), rather than the $\mathrm{n}^{*-4}$ threshold scaling characteristic of static field ionization and high order multiphoton ionization. This altered behavior is due to the short duration and the subcycle nature of the field. We also find a wide distribution of final states produced by these pulses, which are effectively "in resonance" for dozens of transitions simultaneously.

\section{INTRODUCTION}

Single-cycle pulses of freely propagating infrared electromagnetic radiation are novel sources for spectroscopy. These pulses are radiated from biased, large-area GaAs photoconductors illuminated by sub-picosecond laser pulses. We have recently introduced this technique in high-field laser-atom physics. ${ }^{2,3}$ Here we report investigations of photoexcitation and ionization of $\mathrm{Na}$ Rydberg states irradiated by half-cycle pulses. When the pulse length is shorter than the classical Kepler orbit time for these states (the so-called "wavepacket" regime), we find dramatic new phenomena, including coherent redistribution of population in the atom, and a new peak-field ionization threshold law.
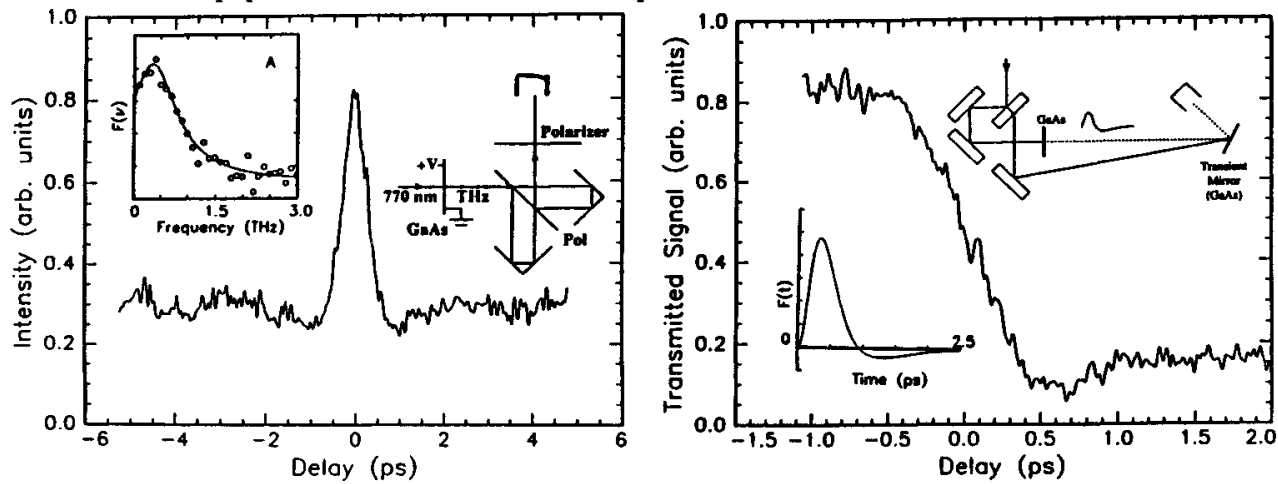

Fig 1. Left: An interferometric field autocorrelation can be used to measure the spectrum of the infrared radiation. ${ }^{2,4}$ The two insets show the derived spectrum, and a block diagram of the apparatus. Right: The pulse duration is determined by transmitting the light through a photoconducting plasma shutter (transient mirror). ${ }^{2,5}$ The upper insert is a diagram of the pump-probe arrangement; the lower inset shows the laser electric field inferred from these two measurements. ${ }^{2}$

\section{GENERATING AND MEASURING HALF-CYCLE PULSES}

The laser used to trigger these pulses is a standard design: a Ti: $\mathrm{Al}_{2} \mathrm{O}_{3}$ self-modelocked oscillator amplified in a Chirped Pulse Amplifier (CPA) ${ }^{6}$ There are two mechanisms responsible for generating the single-cycle pulses: radiation by photocarriers accelerated in the bias electric field ${ }^{7}$; or field- 
induced optical rectification ${ }^{8}$. The former method generally produces a single-cycle radiated field with a large asymmetry in the peak amplitude between the positive and negative lobes -- effectively, a halfcycle pulse. The energy generated depends on the crystal orientation, bias field direction and strength, and the laser polarization, pulselength, and fluence. 'For high bias fields $(>5 \mathrm{kV} / \mathrm{cm})$ on $<100>\mathrm{GaAs}$, we find a fluence of $40 \mu \mathrm{J} / \mathrm{cm}^{2}$ produces the maximum field: a pulse energy of $1 \mu \mathrm{J}$ and peak field in excess of $100 \mathrm{kV} / \mathrm{cm}$, in a nearly unipolar $500 \mathrm{fs}$ electric field pulse. ${ }^{2}$ Techniques used to measure these pulses are described in figure 1.
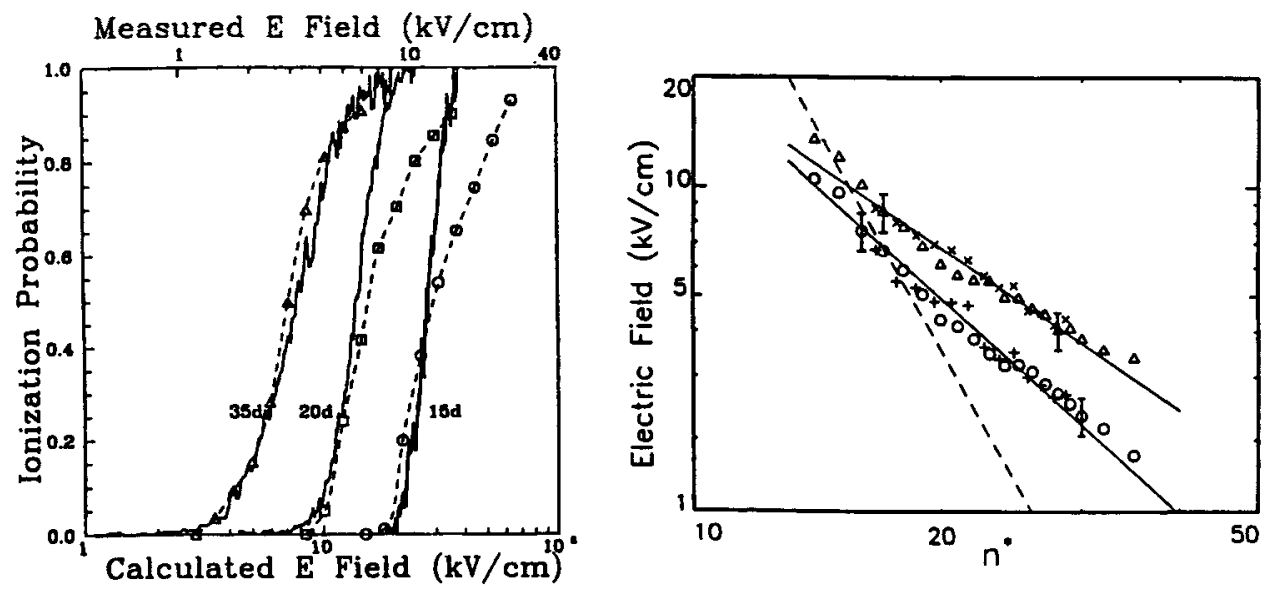

Fig 2. Left: Ionization probability for three Na Rydberg states (35d, 20d, and 15d) as a function of the peak electric field in the half-cycle pulse. ${ }^{3}$ Solid line: data; dotted line: calculation (see text). Right: Compilation of the peak field required for 50\% ionization (upper points) or 10\% ionization (lower points). The solid curves show two scaling behaviors: $F_{\text {crit }} \propto n{ }^{*-3 / 2}$ (upper curve) and $F_{\text {crit }} \propto n^{*-2}$ (lower curve). The dashed curve shows the static field ionization threshold, $\mathrm{F}_{\mathrm{crit}} \propto 1 / 9 \mathrm{n} * 4$.

\section{RYDBERG ATOM IONIZATION}

The largest freely propagating single-cycle pulses are as yet too weak to ionize atomic ground states, but they can easily excite Rydberg states. The bandwidth of the radiation is distributed over about $50 \mathrm{~cm}^{-1}$, with most of the radiation at around $20-30 \mathrm{~cm}^{-1}$. Thus for $\mathrm{n}^{*}>14$, we can expect multiple transitions, as all nearby states can undergo resonant transitions with each other simultaneously. The result might be similar to blackbody excitation, except that here all of the frequency components are coherent.

Figure 2 shows the results of an experiment to measure the ionization fraction of sodium Rydberg states as a function of the peak electric field in the pulse. ${ }^{3}$ Figure 3 contains a diagram of the apparatus. The results show a novel scaling for the field required to ionize a Rydberg state with effective principal quantum number $n *: F_{C R T} \propto 1 / n *^{2}$, not $\propto 1 / n^{* 4}$. A simple classical explanation involves the fact that for a half-cycle pulse, $\int F(t) d t \neq 0$ ! The energy gained by the electron during the pulse is $\int F(t) v(t) d t=\int F(t) v(t) d t$, so ionization is easiest near the core, where the velocity $v(t)$ is large. For high $n$ and low 1 and $m$ states, to a good approximation, $v(t)=\sqrt{2 / x(t)}$. The energy needed to escape is $1 / 2 n{ }^{* 2}$, so the critical field is easily calculated to be $\mathrm{F}_{\mathrm{CRT}} \approx(2 / 5) n^{*-2} \tau_{\mathrm{HCP}}^{-2 / 3}$. This curve is shown passing through the $10 \%$ ionization data in Figure 2.

At the outer edge of the orbit, where the electron is nearly free, it can still gain energy in a halfcycle pulse. A full cycle or many-cycle pulse cannot transfer energy to the outlying (nearly free) electrons. Instead, the strong-field interaction leads to wavepackets of trapped population, so that ionization is incomplete. Our observation of complete ionization, even when the pulse duration is 

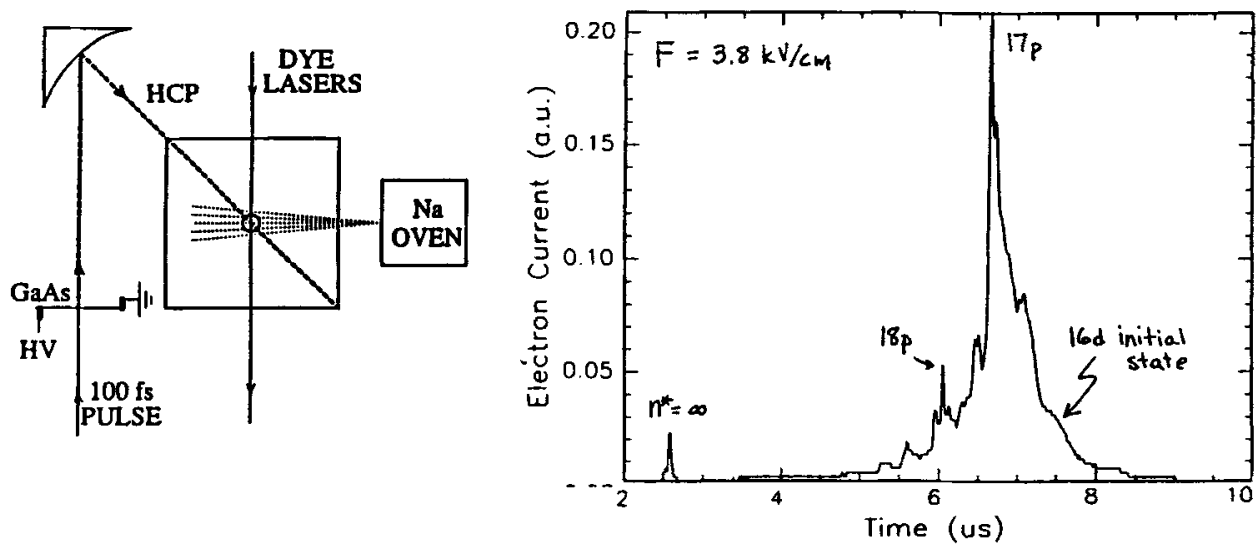

Fig 3 Left: Apparatus for exciting Rydberg states with single-cycle pulses. Right: Single-cycle excitation leads to redistribution of Rydberg population, seen here as additional peaks in a ramped-field state analyzer. In this example, the initial state (16d) is depleted via excitation to states in manifolds with $\mathrm{n}=17,18$, and higher.
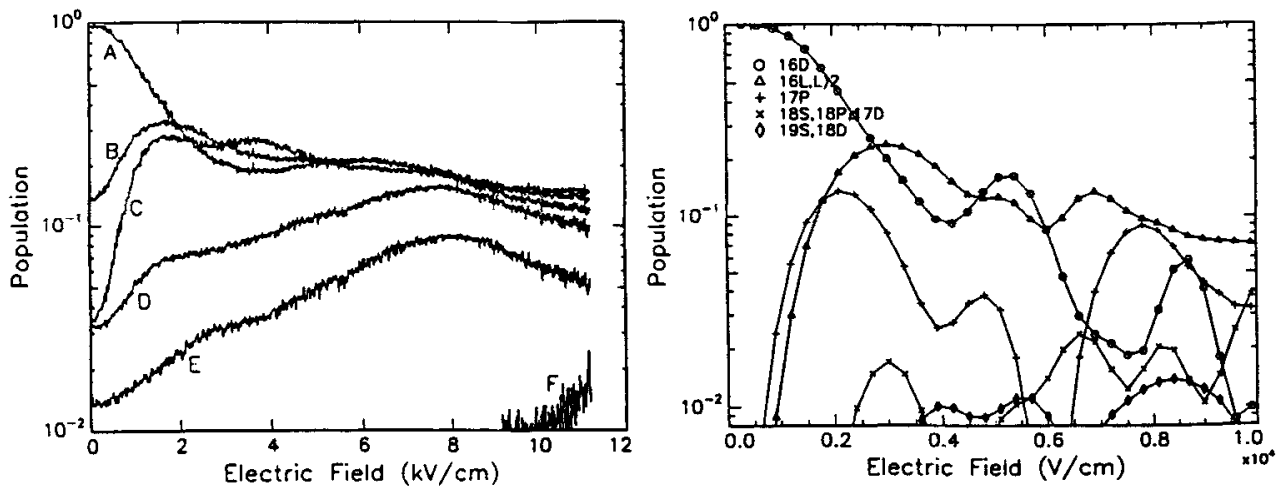

Fig 4 Left: Redistribution of 16d states as a function of peak electric field in the pulse. A: 16d; B: $161,1>2$; C: $17 \mathrm{p}$; D: $18 \mathrm{~s}, 18 \mathrm{p}$, and $17 \mathrm{~d}$ (not individually resolved); E: $19 \mathrm{~s}$ and $18 \mathrm{~d}$; F: ionization. Right: Calculation of the experiment shown at left.

considerably shorter than the Kepler orbit time, is due to the sub-cycle nature of the pulses.

\section{COHERENT POPULATION TRANSFER}

Below the critical field for ionization, population is coherently redistributed by the half-cycle pulse. We analyze the final state distribution with slow ramped-field ionization following the THz pulse (Figure 3). We find that population is typically transferred to dozens of states, including many states which lie outside the bandwidth of the exciting radiation, suggesting multiple transitions or other strong-field effects. The precise distribution is highly sensitive to the pulse length and amplitude, as well as the initial state. Just below $F_{\text {crit }}$, population has typically spread nearly uniformly over dozens of states!

A full quantum calculation of this obviously complicated process is an ambitious task. We have performed a more limited numerical integration of Schroedinger's equation for the atom in a singlecycle field, on a matrix of $74(n, 1)$ states. We find qualitative agreement, as shown in Figure 4 . Certainly,the extreme sensitivity to initial state and to the pulse shape is also evident in our calculation. 


\section{Interactions of Atoms with High-Field Single-Cycle Infrared Radiation}

\section{CONCLUSIONS AND ACKNOWLEDGMENTS}

Our investigations have shown how single-cycle pulses interact with Rydberg atoms. We see new scaling laws and excitation mechanisms which mark the subpicosecond and sub-cycle nature of this novel radiation. We wish to thank D. W. Schumacher and R. Grobe for useful advice and discussions. This work was supported by the National Science Foundation.

\section{REFERENCES}

1. G. Mourou, C. V. Stancampiano, and D. Blumenthal, Appl.Phys.Lett. 38, 470 (1981); D. H. Auston, K. P. Cheung, and P. R. Smith, Appl. Phys. Lett. 45,284 (1984); A. P. DeFonzo, M. Jarwala, and C. Lutz, Appl.Phys.Lett. 50, 1155 (1987); Ch. Fattinger, and D. Grischkowsky, Appl.Phys.Lett. 53, 1480 (1988); P. R. Smith, D. H. Auston, and M. C. Nuss, IEEE J. Quantum Electron. 24, 255 (1988); D. Grischkowsky, Soren Keiding, Martin van Exter, and Ch. Fattinger, J.Opt.Soc.Am.B 7, 2006 (1992).

2. D. You, R. R. Jones, D. R. Dykaar, and P. H. Bucksbaum, Optics Letuers 18, 290 (1993).

3. R. R. Jones, D. You, and P. H. Bucksbaum, Physical Review Letters 70, 1236 (1993).

4. B. I. Greene, J. F. Federici, D. R. Dykaar, R. R. Jones, and P. H. Bucksbaum, Appl.Phys.Lett. 59, 893 (1991).

5. G. Mourou, C. V. Stancampiano, et al., Appl. Phys. Lett. 39, 295 (1981).

6. J. Squier, F. Salin, G. Mourou, and D. Harter, Opt.Lett. 16, 324 (1991).

7. Justin T. Darrow, Xi-Cheng Zhang, David J. Auston, IEEE J. Quantum Electron. 28, 1607 (1992).

8. S. L. Chuang, S. Schmitt-Rink, B. I. Greene, and A. F. J. Levi, Phys.Rev.Lett. 68, 102 (1991); B. I. Greene, P. N. Saeta, D. R. Dykaar, S. Schmidt-Rink, and S. L. Chuang, IEEE Journal of Quantum Electronics Special Issue on Ultrafast Optics and Electronics 28, 2302 (1992).

9. D. You, R. R. Jones, P. H. Bucksbaum, and D. R. Dykaar, submitted to Jour. Opt. Soc. B, (1993). 